\title{
Rethinking protein drug design with highly accurate structure prediction of anti-CRISPR proteins
}

\section{Author Information}

Center for Biosystems and Biotech Data Science, Ghent University Global Campus, Incheon, South Korea

Ho-min Park, Yunseol Park, Joris Vankerschaver, Wesley De Neve, Hyunjin Shim

Department of Electronics and Information Systems, Ghent University, Ghent, Belgium

Ho-min Park, Wesley De Neve

Department of Applied Mathematics, Computer Science and Statistics, Ghent University, Ghent, Belgium

Joris Vankerschaver

Department of Mathematics, University of Liège, Liège, Belgium

Arnout Van Messem

Orcid links

Ho-min Park orcid=0000-0001-9937-8617, Yunseol Park orcid=0000-0002-2005-1847, Joris

Vankerschaver orcid=0000-0002-5813-5659, Arnout $\{$ Van Messem $\}$ orcid=0000-0001-8545-7437,

Wesley $\{$ De Neve $\}$ orcid=0000-0002-8190-3839, Hyunjin Shim orcid=0000-0002-7052-0971

Contributions

Analyses were primarily conducted by H.P., Y.P., and H.S. Specifically, AlphaFold analyses were led by H.P., genetic analyses were performed by Y.P., and structural and functional analyses were conducted by H.S. The study was conceived by H.S., and all authors contributed to writing the manuscript.

Corresponding author

Correspondence to: Hyunjin Shim (iinenstar@gmail.com) 


\footnotetext{
Abstract

Protein therapeutics play an important role in controlling the functions and activities of disease-causing proteins in modern medicine. Despite protein therapeutics having several advantages over traditional small-molecule therapeutics, further development has been hindered by drug complexity and delivery issues. However, recent progress in deep learning-based protein structure prediction approaches such as AlphaFold opens new opportunities to exploit the complexity of these macro-biomolecules for highly-specialised design to inhibit, regulate or even manipulate specific disease-causing proteins. Anti-CRISPR proteins are small proteins ( $<100$ amino acids) from bacteriophages that counter-defend against the prokaryotic adaptive immunity of CRISPR-Cas systems. They are unique examples of natural protein therapeutics that have been optimized by the host-parasite evolutionary arms race to inhibit a wide variety of host proteins. Here, we show that these Anti-CRISPR proteins display diverse inhibition mechanisms through accurate structural prediction and functional analysis. We find that these phage-derived proteins are extremely distinct in structure, some of which have no homologues in the current protein structure domain. Furthermore, we find a novel family of Anti-CRISPR proteins which are structurally homologous to the recently-discovered mechanism of manipulating host proteins through enzymatic activity, rather than through direct inference. Using highly accurate structure prediction, we present a wide variety of protein-manipulating strategies of anti-CRISPR proteins for future protein drug design.
} 


\section{Introduction}

Proteins are macromolecules composed of amino-acid residues that perform diverse roles in biological entities, including catalysing biochemical reactions, providing cell/capsid structure, transporting molecules, replicating genetic material and responding to stimuli. It is estimated there are over 25,000 functionally distinct proteins in the human body ${ }^{1,2}$, and mutations or abnormalities in these proteins may result in diseases. Thus, modern medicine has focused on harnessing target proteins to alleviate diseases, mostly through small-molecule therapeutic agents acting as competitive or noncompetitive inhibitors ${ }^{3}$. However, it is estimated that only $\sim 10 \%$ of the human proteome can be targeted with small-molecule drugs $^{3}$. Since the introduction of human insulin as the first recombinant protein therapeutic in the $1980 \mathrm{~s}^{4,5}$, protein-based therapeutics are expanding the scope of "druggable proteins". Compared to small-molecule drugs, the major advantage of protein therapeutics is improved target specificity and reduced immunogenicity due to their proteinaceous nature ${ }^{5}$. Protein therapeutics can also serve complex functions that simple chemical compounds cannot achieve, such as replacing a deficient protein or providing a protein of novel function (Fig. 1a). Furthermore, protein therapeutics can inhibit disease-related proteins that small-molecule drugs cannot target due to the lack of a cavity to bind. Currently, there are over 130 protein therapeutics commercially available and intense research efforts are ongoing to better design protein therapeutics ${ }^{5}$.

In this study, we present a group of naturally-occurring protein therapeutics called anti-CRISPR (Acr) proteins, as a prominent example of how small proteins are used by invading bacteriophages (phages) in nature to control host proteins. Phages are the most abundant and diverse biological entities in the biosphere (estimated $10^{31}$ phages) that infect and replicate within host prokaryotes (such as bacteria or archaea) ${ }^{6}$. High selective pressures between these parasites and hosts drive dynamic coevolution of genomic and proteomic mechanisms and systems ${ }^{7-9}$. In particular, the evolutionary arms race between 
phages and prokaryotes has resulted in a vast arsenal of immune systems, including the prokaryotic adaptive immune system known as CRISPR-Cas ${ }^{10,11}$. CRISPR-Cas systems are defence mechanisms against phages (and other mobilomes) through a complex of RNA-guided Cas proteins (Fig. 1b). Remarkably, prokaryotic genomes with CRISPR-Cas systems can acquire short fragments of foreign genetic sequences in their CRISPR arrays, which serve as RNA templates to recognize and cleave invading phages through the nuclease complex of Cas proteins ${ }^{12}$. Since the successful application of CRISPR-Cas systems as genome-editing tools ${ }^{13,14}$, there was a burst in the discovery of diverse CRISPR-Cas systems ${ }^{15}$, followed by the discovery of Acr proteins that neutralize the activity of this prokaryotic adaptive immune system ${ }^{16}$ (Fig. 1b). A family of Acr proteins was first identified in the CRISPR-Cas-inactivating prophages of Pseudomonas genomes that disable Type I-F and Type I-E CRISPR-Cas systems ${ }^{16,17}$. A number of Acr proteins inhibiting type II CRISPR-Cas systems have since been applied as regulators of gene-editing activities ${ }^{18}$. Acr protein families are known to have short sequences ( $<100$ amino acids) with no common genetic features, and interact directly with Cas proteins to inhibit target DNA binding, DNA cleavage, CRISPR RNA loading and protein-complex formation ${ }^{18,19}$. A recent study reveals AcrVA5 proteins to inactivate Cas12a of Type V CRISPR-Cas systems enzymatically by acetylation of the active site, with structural homology to an acetyltransferase protein ${ }^{20}$.

In this study, we conduct a comprehensive analysis on the key characteristics of Acr proteins viewed from the perspective of naturally-occurring protein therapeutics that effectively inhibit host protein functions. Motivated by the observation that these Acr proteins are genetically diverse, we examine the protein structure of these diverse proteins using AlphaFold ${ }^{21}$. AlphaFold is a state-of-the-art deep learning-based approach that performs protein structure prediction, which takes a protein sequence as an input to predict its 3-D protein structure through an iterative exchange of information between its genetic representation and its structural representation. The recent release of AlphaFold, the winner of CASP14 which achieves highly accurate protein structure predictions ${ }^{21,22}$, is revolutionary for the field of 
life sciences and medicine, and it is expected to accelerate critical research in a large number of fields ranging from structural biology to drug discovery. In this study, we first assess the performance of AlphaFold in predicting the 3-D structures of Acr proteins based on similarity metrics against their experimentally reconstructed 3-D macromolecular structures. Using this performance as a basis, we further examine the Acr proteins without experimental structures with AlphaFold, to predict the structural diversity of these genetically distinct proteins that are natural inhibitory proteins against prokaryotic CRISPR-Cas systems. We use AlphaFold-predicted structures of Acr proteins to infer a range of inhibition mechanisms through homology search and functional analysis, to demonstrate how bacteriophages exploit diverse strategies to manipulate host immune systems, with the long-term goal of providing an unique opportunity to learn from the evolution-optimized inhibitor proteins for future protein drug design. 


\section{Results}

\section{AlphaFold prediction of anti-CRISPR protein structures}

Acr protein datasets were acquired from various viral and prokaryotic genomes, including Pseudomonas

phage, Pseudomonas aeruginosa and Escherichia coli ${ }^{23}$, which were categorised into three sets: verified Acr proteins with experimental structure (Set A), verified Acr proteins without experimental structure

(Set B), and putative Acr proteins with experimental structure (Set C) (Supplementary Table 1). From the AlphaFold-predicted structures of each set (Fig. 2a), we compared the prediction performance between CASP14 (52 AlphaFold evaluation results from the CASP14 competition), Set A and Set C (Fig. 2b and 2c), based on TM-scores and relative Z-errors (see Methods for details) against the true experimental structures, where Set B without experimental structures was excluded (Supplementary Table 2).

According to the TM-score, CASP14 had a higher median than Set A and Set C (0.925 vs. 0.895; 0.843, respectively), but Set A had the highest mean as compared to Set C and CASP14 (0.896 vs. 0.868; 0.882, respectively). Furthermore, Set A and Set C had significantly smaller standard deviations than CASP14 $(0.095 ; 0.092$, respectively, vs. 0.120$)$, indicating that, according to the TM-score, their predictions are more accurate. According to the relative Z-error, CASP14 recorded a lower median than Set A and Set C (0.201 vs. $0.217 ; 0.230$, respectively), but Set A recorded the lowest mean as compared to CASP14 and Set C (0.211 vs. $0.237 ; 0.259$, respectively). Similarly to the TM-score, Set A and Set C recorded smaller standard deviations than CASP14 $(0.128 ; 0.129$, respectively, vs. 0.141). As can be seen in the boxplots (Fig. $2 \mathrm{~b}$ and 2c), there was no significant difference in prediction performance, validating that AlphaFold predicts 3-D structures of Acr proteins as accurately as the CASP14 dataset. 


\section{Evolutionary trees of Anti-CRISPR proteins}

Acr proteins are known to be genetically diverse; this raises an intriguing question about the origin and evolution of Acr proteins. We reconstructed evolutionary trees of the verified Acr proteins (Set A + Set B; $n=207$ ), using sequence-based and structure-based methods (see Methods for details). As expected, the phylogenetic tree built using genetic sequences (Fig. 3a) shows high levels of variation, while forming consistent clades with the high bootstrap support. Analysis of the clades reveals some degrees of clustering by the Acr family at the shallower nodes; however, this clustering is mostly due to the near-identical protein sequences. For instance, many of the AcrIIA proteins were derived from AcrIIA1 and AcrIIA2, driven by the technological interest to regulate CRISPR-Cas gene-editing activities in different cell types ${ }^{18}$. Otherwise, the phylogenetic tree shows absence of clustering by other biological features such as taxonomy and inhibition mechanism.

Given the low sequence similarity among the Acr proteins, we built a structure-based tree using AlphaFold-predicted structures, which includes 98 Acr proteins without experimental structures (Fig. 3b). The structural tree shows an even higher level of diversity in the Acr proteins than the phylogenetic tree. In the structural tree, the Acr proteins share no common ancestor and display deep branches, consistent with earlier observations of how evolutionary pressure drives immunity-related mechanisms of hosts and parasites to coevolve rapidly ${ }^{8,9}$. The structural tree also shows some degrees of clustering by the Acr family, but the clusters do not always coincide between the two evolutionary trees. From the visual analysis of the protein structures, the branches of the structural tree are placed randomly in terms of structural forms and the functions are only related at the clade level (Supplementary Fig. 1).

It is evident that the sequence-based and structure-based trees capture different evolutionary relationships between the Acr proteins. It is known that the 3-D structures of homologous proteins are better conserved than their corresponding genetic sequences, particularly when the sequence similarity is 
below $30 \%{ }^{24}$. From the multiple sequence alignment, no site of the Acr proteins is conserved at $30 \%$ and only very few sites are conserved at 15\% (Supplementary Fig. 2). We calculated the congruence among distance matrices of the sequence-based and structure-based trees to be very low according to the measure of congruence (Kendall's coefficient of concordance, $\mathrm{W}=6.58 \mathrm{e}-01$ ), confirming the correlation between these two types of evolutionary trees is poor among highly divergent proteins ${ }^{24}$.

\section{Structural homology to anti-CRISPR predicted structures}

Characterised Acr proteins use diverse strategies to interfere directly with CRISPR-Cas systems, including inhibiting DNA binding, DNA cleavage, guide loading and ribonuclease activity ${ }^{18}$. To investigate the relation between 3-D protein structures and inhibitory functions, we identified homologous structures to the AlphaFold-predicted Acr proteins using structure-based distance measures ${ }^{25}$ (see Methods for details).

First, we used a subset of the Acr dataset with experimental structures (Set A) to successfully validate the closest structural homologue to each AlphaFold-predicted Acr protein matched with its true experimental structure (Supplementary Table 3).

Second, we analyzed the Acr proteins without experimental structures (Set B) by matching the AlphaFold-predicted structures to the closest homologues from the Protein Data Bank archive ${ }^{25}$ (Supplementary Table 4). Functional analysis of the closest homologue to each Acr protein reveals a wide variety of protein functions, including polymerase, ligase, nuclease, regulation, and transport (Fig. 4c). We acknowledge that some of the closest homologues have low structural similarity (Z-score below 4); however, it is intriguing that these Acr proteins have no close structural homologues in the Protein Data Bank. Some Acr proteins from the families AcrIC6, AcrVIA2, AcrIIA19, and AcrIF8 ( Fig. 4a) have no structural homologues (significance threshold for similarity: Z-score $=2$ ). In addition, we validated that 
Acr proteins retrieved their neighbours in the same clade as the closest homologue, when their neighbouring proteins had experimental structures in the Protein Data Bank. For example, the Acr proteins labelled '0434' and '0435' are in the same clade, and the closest homologue of the Acr protein ' 0435 ' matched with the experimental structure of the Acr protein ' 0434 '.

Third, we cross-examined the Acr proteins whose inhibitory mechanism was experimentally characterised to verify that the homologues retrieved were functionally related (indicated as the middle ring in Fig. 3b). The homologue functions of this subset were related to a wide variety of functional domains (Supplementary Table 4). For instance, the two Acr proteins in the same clade (labelled '3628' and ' 3642 ') were characterised to inhibit the DNA-binding of Cas proteins ${ }^{26}$ and their structural homologues have functional domains of ligase. A few closest homologues with functions related to acetyltransferase drew particular attention, as a recent biochemical study revealed an unprecedented mechanism of inhibiting CRISPR-Cas systems through enzymatic activity rather than through direct interaction ${ }^{20}$. According to this study, the closest structural homologue to this Acr protein (labelled '3625') was found to be N-Alpha-Acetyltransferase (4U9W-C) (Fig. 4b). We found another homologue (1Y9W-A) with a better similarity score to the AlphaFold-predicted structure of this Acr protein, with the functional annotation of acetyltransferase (Table 1). Furthermore, we found a number of uncharacterised Acr proteins in the same clade of the structural tree (between '0430' and '3681') related to Acetylglucosaminidase from various Acr families, including AcrIC, AcrIE, AcrIF, AcrIIA, and AcrVIB. Intriguingly, several proteins have homologues with the functional annotations of nuclease activity, which is reminiscent of the newly-discovered mechanism of nuclease activity against crRNAs and CRISPR-Cas signalling molecules ${ }^{27,28}$. 


\section{New anti-CRISPR family of acetylation inhibition}

A recent study reveals a new Acr protein that disables Type V Cas12a by acetylation, which leads to a complete loss of the DNA-cleavage activity ${ }^{20}$. Surprisingly, this Acr protein (labelled '3625') has been found to structurally align closest to an amino-terminal acetyltransferase (4U9W-C) (Fig. 4b), despite their low sequence similarity. We identified other Acr proteins that are related to this AcrVA5 protein on the evolutionary trees of the Acr proteins (Fig. 3b). Notably, there are two Acr proteins in the same clade as the AcrVA5 protein on the structural tree, one of which was lacking experimentally validated structure or function. Analysis of its function through the structural homologues reveals that this Acr protein (labelled '3666') is related to acetyltransferase. Interestingly, this Acr protein belongs to a different Acr family (AcrIB) than the previously identified AcrVA5. Its superimposition with the closest structural homologue reveals a similar structural alignment at the functionally critical site of the acetyltransferase where acetyl-CoA binds (Fig. 4b). The sequence identity of the AcrIB protein to its closest homologue was found to be $17.6 \%$, while the structural identity between these two proteins in 3-D was found to be higher at 21\% (Table 1 and Supplementary Fig. 3). On the phylogenetic tree, this AcrIB protein is not placed close to the other two Acr proteins of acetyltransferase function (labelled '3625' and '0557') (Fig. 3a), demonstrating that these two types of Acr proteins have close structural similarity but not genetic similarity. This finding suggests that for proteins with low sequence similarity, structure-based trees cluster proteins with most similar biochemical functional properties better than sequence-based trees ${ }^{24}$. Using the structural tree, we discovered a new family of Acr proteins belonging to AcrIB that had a strong structural homology to acetyltransferase from a different organism (gram-negative bacteria Salmonella enterica), whereas the previously characterised AcrVA5 matched to acetyltransferase from gram-positive bacteria Bacillus cereus. 


\section{Discussion}

We show that the 3-D structures of Acr proteins predicted with AlphaFold achieve high accuracy. The structural tree reconstructed from these AlphaFold-predicted structures show more diversity of Acr proteins with no common evolutionary origin as compared to the phylogenetic tree. On the structural tree, the Acr proteins form small clades by the unique structural similarity, which are also related by the inhibition mechanism. The functional annotations of the Acr protein homologues are extremely diverse, including a wide range of enzymatic and regulatory activities from different organisms. Most characterised Acr proteins inhibit host CRISPR-Cas systems by direct interference; we show that this category of Acr proteins displays various functional annotations and unique structural forms in the multiple branches of the structural tree.

In particular, we found a number of Acr proteins with homologue annotations related to acetylation. A recent discovery of Acr proteins that manipulate CRISPR-Cas systems through enzymatic activities demonstrates extensive phage defence mechanisms driven by the intense host-parasite arms race 20,27. Through the AlphaFold-predicted structural analysis, we found a novel family of Acr proteins (AcrIB) from the genome of a human pathogen (Leptotrichia buccalis $\mathrm{C}-1013-\mathrm{b})$ that shows more structural similarity to acetyltransferase than the previously characterised AcrVA protein. Intriguingly, other Acr proteins on the multiple branches of the structural tree have homologues related to different types of acetyltransferase enzymes from heterologous species. Acr proteins could evolve independently from various host genomes and mobile genetic elements, exploiting a vast inventory of protein structures as the basis for their counter-defence advantage ${ }^{6,9,29}$.

More broadly, Acr proteins are exceptional examples of coevolution dynamics optimizing the phage genomes to manipulate host systems and maximize survival. Since phages can only replicate within host cells and are void of metabolic capacity to synthesize small molecules, their counter-defence machinery against the sophisticated and extensive prokaryotic anti-phage systems is protein-based. 
Nonetheless, phages are the most abundant organisms in the biosphere ${ }^{30}$, and their successful protein-based viral arsenals such as Acr proteins provide an important insight on how to expand the potential of protein therapeutics against disease-causing proteins. Specifically, we could get inspiration from these phage-derived protein structures that resemble segments of related or target proteins, to design highly-specialised protein inhibitors with diverse protein-manipulating strategies, including indirect interference through enzymatic activities ${ }^{31}$. The high biodegradability issue of protein therapeutics has partially been solved by the recent success of mRNA vaccine delivery using lipid nanoparticles ${ }^{32}$, making low risk protein therapeutics ever more attractive to the industry. From the AlphaFold-predicted structures, we accelerated the structural and functional analysis of Acr proteins whose experimental 3-D structures are yet to be resolved. Given the number of Acr proteins without homologues in the current protein structure domain, we wonder whether there is a vast repertoire of unexplored protein structural configurations that can be exploited for protein drug design. 


\section{Methods}

\section{Curation of anti-CRISPR datasets}

The Anti-CRISPR dataset contains 446 Acr proteins (210 verified, 236 putative) that inhibit a wide range of CRISPR-Cas systems including I, II, III and VI from the Anti-CRISPRdb ${ }^{23}$. The term 'verified' indicates the protein was validated as CRISPR-Cas-inactivating Acr (either by the database or other published papers), while 'putative' indicates the protein was predicted to be Acr without sufficient experimental support. The Anti-CRISPR dataset was further curated into Set A, Set B, and Set C for AlphaFold, according to the availability of experimentally reconstructed 3-D macromolecular structures (hereby referred to as "experimental structures") (Supplementary Table 1). Each protein is annotated with Acr family, type of inhibited CRISPR-Cas systems, NCBI accession, genetic sequence, source organism, taxonomy, and inhibitory mechanism when available (Supplementary Tables 3 and 4).

\section{Prediction of anti-CRISPR protein structure with AlphaFold}

We predicted the 3-D protein structures of each set with AlphaFold, using the Acr protein sequence as the input to AlphaFold (Fig. 2a). AlphaFold creates genetic and structure representations by comparing the protein sequence with several pre-installed databases. Those representations are used as input to five prediction models to generate five candidate 3-D structures. The result with the highest per-residue confidence score (pLDDT: per residue estimate of confidence on a scale from 0 to $100^{33}$ ) among the five results is determined as the final structure and saved in a protein data bank (PDB) format (Supplementary Fig. 4). For the Acr datasets, we used the PDB archive until 31/12/2012 as templates in AlphaFold to exclude the true experimental structures of the Acr proteins. The details of the experiments related to the 
hardware specification and to the processor performance are given in the Supplementary material (Supplementary Table 5 and Supplementary Table 6, respectively).

\section{Comparison of AlphaFold performance on anti-CRISPR against CASP14}

To validate the performance of AlphaFold on predicting Acr structures, we benchmarked the CASP14 dataset against Set $\mathrm{A}$ and Set $\mathrm{C}$ of Acr proteins with the corresponding true experimental structures available. We excluded predicted structure and experimental structure pairs for which the TM-score and/or the Z-score were too low. Finally, 52 pairs of CASP14, 99 pairs of Set A, and 207 pairs of Set C were used for the comparison study. We used the TM-score ${ }^{34,35}$ and Dali Z-score ${ }^{36}$ as similarity measures between the predicted and the experimental structures. Unlike traditional metrics (e.g. root-mean-square deviation), the TM-score is length-independent and more sensitive to the global similarity than to the local variation. The Dali Z-score is the sum of the equivalent residue-wise intermolecular distances among two proteins, and does not have a fixed upper bound ${ }^{36}$. We then used the following relative Z-error to calculate the relative difference:

$$
Z_{\text {error }}=\frac{Z_{g t}-Z_{p d}}{Z_{g t}}
$$

where $Z_{g t}$ is the self Dali Z-score between experimental structure and itself, and $Z_{p d}$ is the Dali Z-score between experimental structure and predicted structure. We obtained the $Z_{p d}$ and TM-score for the CASP14 set of AlphaFold from the CASP14 assessment scores ${ }^{37}$, whereas $Z_{g t}$ of CASP14 and $Z_{g t}$ and $Z_{p d}$ of Set A and Set $\mathrm{C}$ were calculated using DaliLite.v5 ${ }^{36}$. Finally, a protein structure comparison and clustering tool called MaxCluster ${ }^{38}$ was used to calculate the TM-scores of Set A and Set C. Both 
distance metrics have values between 0 and 1 , with 1 as the best score for TM-scores and 0 as the best score for relative Z-errors.

\section{Reconstruction of evolutionary trees of anti-CRISPR proteins}

We reconstructed the evolutionary trees of the Anti-CRISPR dataset (Set A and Set B) using sequence-based and structure-based inference. The sequence-based tree of the Acr proteins was built by aligning the amino acid sequences using a multiple alignment program, MAFFT (version 7.471, -auto option) ${ }^{39}$. The multiple sequence alignment of the Acr proteins was then visualized using Jalview (version 2.11.1.3) with a conservation visibility of $15 \%$ (Supplementary Fig. 2$)^{40}$. Subsequently, a phylogenetic tree of the Acr proteins was built with IQ-Tree using ModelFinder (-auto option) to find the best-fit model among the supported range of protein substitution models ${ }^{41,42}$ (Supplementary Table 7). Using the best-fit substitution model, 1,000 ultrafast bootstrap replicates were run to check bootstrap support of the reconstructed tree topology ${ }^{43}$.

The structure-based tree of the Acr proteins was built by calculating the similarity matrix between the Dali Z-scores of the AlphaFold-predicted structures and its corresponding experimental structures. We used the Dali server ${ }^{36}$ for generating structural trees from hierarchical clustering of the similarity matrix. The structural tree of the Acr proteins was generated from distance matrices, where the pseudo-distance between two structures $\mathrm{Q}$ and $\mathrm{T}$ was defined as ${ }^{44}$ :

$$
D_{Q T}=Z_{Q Q}+Z_{T T}-2 Z_{Q T}
$$

The hierarchical clustering of the similarity matrix was outputted as a Newick formatted dendrogram. The phylogenetic tree and the structural tree of the Acr proteins were visualized with iTOL (version 4) and iTOL annotation editor ${ }^{45,46}$ with the following labels: Acr Family, Taxonomy, and Inhibition Mechanism. 


\section{Congruence among distance matrices of sequence-based and structure-based trees}

We measured the congruence among distance matrices of the reconstructed trees from the sequence-based and structure-based methods using Kendall's coefficient of concordance, W, which ranges from 0 (no congruence) to 1 (complete congruence) ${ }^{47}$. First, we computed the cophenetic value of pairwise distances between the terminals from a phylogenetic tree using its branch lengths with the function cophenetic.phylo from ape-package (version 5.0) ${ }^{48}$. Then, we used the function CADM.global to calculate the coefficient of concordance among the distance matrices of the sequence-based and structure-based trees of the Acr proteins through a permutation test.

\section{Visualization of protein structure superimposition}

For functional analysis, AlphaFold-predicted structures with functional annotations of interest were superimposed with their structural homologues using PyMol (version 2.5.2) to visualize the overlap in structure of the functionally active sites. The inhibitory mechanism of Acr proteins without experimental structure was inferred through examining functional annotations of the structural homologues to the AlphaFold-predicted structure, with the significance threshold of Z-score $>4$. 


\section{Data Availability}

All the input protein sequences are available in Anti-CRISPRdb (https://guolab.whu.edu.cn/anti-CRISPRdb) as well as our project GitHub page (https://github.com/powersimmani/ACR alphafold). The 3-D structures from the Protein Data Bank were used as ground truth for calculating Dali Z-scores and TM-scores; and superimposing structures (downloaded on 13/10/2021). All AlphaFold-predicted 3-D structures for Acr proteins are available on our github project page, including 3-D images. TM-scores and Dali Z-scores of AlphaFold; ground truths of CASP14 were acquired from the CASP (Critical Assessment of Structure Prediction) competition (https://predictioncenter.org/casp14).

\section{Code Availability}

Protein structures were predicted with AlphaFold, available under an open-source license at https://github.com/deepmind/alphafold. For protein structure similarity metrics, we used MaxCluster (http://www.sbg.bio.ic.ac.uk/ maxcluster/index.html) for TM-score and DaliLite.v5 (http://ekhidna2.biocenter.helsinki.fi/dali/README.v5.html) for Dali Z-score. For MSA, we used MAFFT.v7 (https://mafft.cbrc.jp/alignment/server) and Jalview.v2 (https://www.jalview.org) for visualization. For phylogenetic tree reconstruction, we used IQ-Tree (http://www.iqtree.org) with ModelFinder and UFBoot options. For structural tree reconstruction, we used Dali server (http://ekhidna2.biocenter.helsinki.fi/dali) for building dendrograms. 3-D Structure visualizations were created in Pymol v.2.5.2 (https://pymol.org) and Py3DMol v.1.7.0 (https://pypi.org/project/py3Dmol) with Jupyter v.1.0.0 (https://jupyter.org). For data analysis, Python .3.6.4 (https://www.python.org), NumPy v.1.17.5 (https://github.com/numpy/numpy), SciPy v.1.1.0 (https://www.scipy.org), seaborn v.0.9.0 (https://github.com/mwaskom/seaborn), Matplotlib v.3.3.4 (https://github.com/matplotlib/matplotlib), pandas v.0.22.0 (https://github.com/pandas-dev/pandas) were used.

\section{Acknowledgements (optional)}

The research and development activities described in this study were funded by Ghent University Global Campus (GUGC), Incheon, Korea.

\section{Competing interests}

None

\section{Supplementary Information}

Supplementary Information will be provided upon request. 


\section{References}

1. Initial sequencing and analysis of the human genome. Nature 409, 860-921 (2001).

2. The Sequence of the Human Genome. Science vol. 291 1155.4-1155 (2001).

3. Hopkins, A. L. \& Groom, C. R. The druggable genome. Nature Reviews Drug Discovery vol. 1 727-730 (2002).

4. Goeddel, D. V. et al. Expression in Escherichia coli of chemically synthesized genes for human insulin. Proceedings of the National Academy of Sciences vol. 76 106-110 (1979).

5. Leader, B., Baca, Q. J. \& Golan, D. E. Protein therapeutics: a summary and pharmacological classification. Nat. Rev. Drug Discov. 7, 21-39 (2008).

6. Dion, M. B., Oechslin, F. \& Moineau, S. Phage diversity, genomics and phylogeny. Nat. Rev. Microbiol. 18, 125-138 (2020).

7. Koonin, E. V., Dolja, V. V. \& Krupovic, M. Origins and evolution of viruses of eukaryotes: The ultimate modularity. Virology 479-480, 2-25 (2015).

8. Watson, B. N. J., Steens, J. A., Staals, R. H. J., Westra, E. R. \& van Houte, S. Coevolution between bacterial CRISPR-Cas systems and their bacteriophages. Cell Host Microbe 29, 715-725 (2021).

9. Hampton, H. G., Watson, B. N. J. \& Fineran, P. C. The arms race between bacteria and their phage foes. Nature 577, 327-336 (2020).

10. Jansen, R., van Embden, J. D. A., Gaastra, W. \& Schouls, L. M. Identification of genes that are associated with DNA repeats in prokaryotes. Mol. Microbiol. 43, 1565-1575 (2002).

11. Mojica, F. J. M., Díez-Villaseñor, C., García-Martínez, J. \& Soria, E. Intervening sequences of regularly spaced prokaryotic repeats derive from foreign genetic elements. J. Mol. Evol. 60, 174-182 (2005).

12. Barrangou, R. et al. CRISPR provides acquired resistance against viruses in prokaryotes. Science 315, 1709-1712 (2007).

13. Deltcheva, E. et al. CRISPR RNA maturation by trans-encoded small RNA and host factor RNase III. Nature 471, 602-607 (2011).

14. Jinek, M. et al. A programmable dual-RNA-guided DNA endonuclease in adaptive bacterial immunity. Science 337, 816-821 (2012).

15. Makarova, K. S. et al. Evolutionary classification of CRISPR-Cas systems: a burst of class 2 and derived variants. Nat. Rev. Microbiol. 18, 67-83 (2020).

16. Bondy-Denomy, J., Pawluk, A., Maxwell, K. L. \& Davidson, A. R. Bacteriophage genes that inactivate the CRISPR/Cas bacterial immune system. Nature 493, 429-432 (2013).

17. Pawluk, A., Bondy-Denomy, J., Cheung, V. H. W., Maxwell, K. L. \& Davidson, A. R. A new group of phage anti-CRISPR genes inhibits the type I-E CRISPR-Cas system of Pseudomonas aeruginosa. MBio 5, e00896 (2014).

18. Marino, N. D., Pinilla-Redondo, R., Csörgő, B. \& Bondy-Denomy, J. Anti-CRISPR protein applications: natural brakes for CRISPR-Cas technologies. Nature Methods vol. 17 471-479 (2020).

19. Pawluk, A. et al. Inactivation of CRISPR-Cas systems by anti-CRISPR proteins in diverse bacterial species. Nat Microbiol 1, 16085 (2016).

20. Dong, L. et al. An anti-CRISPR protein disables type V Cas12a by acetylation. Nat. Struct. Mol. Biol. 26, 308-314 (2019).

21. Jumper, J. et al. Highly accurate protein structure prediction with AlphaFold. Nature 596, 583-589 (2021).

22. Callaway, E. 'It will change everything': DeepMind's AI makes gigantic leap in solving protein structures. Nature 588, 203-204 (2020).

23. Dong, C. et al. Anti-CRISPRdb: a comprehensive online resource for anti-CRISPR proteins. Nucleic 
Acids Res. 46, D393-D398 (2017).

24. Balaji, S. \& Srinivasan, N. Comparison of sequence-based and structure-based phylogenetic trees of homologous proteins: Inferences on protein evolution. J. Biosci. 32, 83-96 (2007).

25. Berman, H. M. et al. The Protein Data Bank. Nucleic Acids Res. 28, 235-242 (2000).

26. Watters, K. E. et al. Potent CRISPR-Cas9 inhibitors from genomes. Proc. Natl. Acad. Sci. U. S. A. 117, 6531-6539 (2020).

27. Athukoralage, J. S. et al. An anti-CRISPR viral ring nuclease subverts type III CRISPR immunity. Nature 577, 572-575 (2020).

28. Knott, G. J. et al. Broad-spectrum enzymatic inhibition of CRISPR-Cas12a. Nat. Struct. Mol. Biol. 26, 315-321 (2019).

29. Shim, H. Feature Learning of Virus Genome Evolution With the Nucleotide Skip-Gram Neural Network. Evol. Bioinform. Online 15, 1176934318821072 (2019).

30. Frost, L. S., Leplae, R., Summers, A. O. \& Toussaint, A. Mobile genetic elements: the agents of open source evolution. Nat. Rev. Microbiol. 3, 722-732 (2005).

31. Shim, H., Shivram, H., Lei, S., Doudna, J. A. \& Banfield, J. F. Diverse ATPase Proteins in Mobilomes Constitute a Large Potential Sink for Prokaryotic Host ATP. Front. Microbiol. 12, 691847 (2021).

32. Jackson, N. A. C., Kester, K. E., Casimiro, D., Gurunathan, S. \& DeRosa, F. The promise of mRNA vaccines: a biotech and industrial perspective. NPJ Vaccines 5, 11 (2020).

33. Tunyasuvunakool, K. et al. Highly accurate protein structure prediction for the human proteome. Nature 596, 590-596 (2021).

34. $\mathrm{Xu}, \mathrm{J}$. \& Zhang, Y. How significant is a protein structure similarity with TM-score $=0.5$ ? Bioinformatics vol. 26 889-895 (2010).

35. Zhang, Y. \& Skolnick, J. Scoring function for automated assessment of protein structure template quality. Proteins: Structure, Function, and Bioinformatics vol. 68 1020-1020 (2007).

36. Holm, L. Using Dali for Protein Structure Comparison. Methods Mol. Biol. 2112, 29-42 (2020).

37. Kryshtafovych, A., Schwede, T., Topf, M., Fidelis, K. \& Moult, J. Critical assessment of methods of protein structure prediction (CASP)-Round XIV. Proteins (2021) doi:10.1002/prot.26237.

38. Siew, N., Elofsson, A., Rychlewski, L. \& Fischer, D. MaxSub: an automated measure for the assessment of protein structure prediction quality. Bioinformatics 16, 776-785 (2000).

39. Katoh, K., Rozewicki, J. \& Yamada, K. D. MAFFT online service: multiple sequence alignment, interactive sequence choice and visualization. Brief. Bioinform. 20, 1160-1166 (2019).

40. Taylor, W. R. Residual colours: a proposal for aminochromography. Protein Engineering Design and Selection vol. 10 743-746 (1997).

41. Nguyen, L.-T., Schmidt, H. A., von Haeseler, A. \& Minh, B. Q. IQ-TREE: a fast and effective stochastic algorithm for estimating maximum-likelihood phylogenies. Mol. Biol. Evol. 32, 268-274 (2015).

42. Kalyaanamoorthy, S., Minh, B. Q., Wong, T. K. F., von Haeseler, A. \& Jermiin, L. S. ModelFinder: fast model selection for accurate phylogenetic estimates. Nat. Methods 14, 587-589 (2017).

43. Hoang, D. T., Chernomor, O., von Haeseler, A., Minh, B. Q. \& Vinh, L. S. UFBoot2: Improving the Ultrafast Bootstrap Approximation. Mol. Biol. Evol. 35, 518-522 (2018).

44. Holm, L. DALI and the persistence of protein shape. Protein Science vol. 29 128-140 (2020).

45. Yang, Z. A space-time process model for the evolution of DNA sequences. Genetics 139, 993-1005 (1995).

46. Letunic, I. \& Bork, P. Interactive Tree Of Life (iTOL) v4: recent updates and new developments. Nucleic Acids Res. 47, W256-W259 (2019).

47. Legendre, P. Species associations: the Kendall coefficient of concordance revisited. Journal of Agricultural, Biological, and Environmental Statistics vol. 10 226-245 (2005). 
48. Paradis, E. \& Schliep, K. ape 5.0: an environment for modern phylogenetics and evolutionary analyses in R. Bioinformatics 35, 526-528 (2019).

49. Bishop, B. et al. Crystal structures of four types of human papillomavirus L1 capsid proteins: understanding the specificity of neutralizing monoclonal antibodies. J. Biol. Chem. 282, 31803-31811 (2007).

50. Fávero-Retto, M. P., Palmieri, L. C., Souza, T. A. C. B., Almeida, F. C. L. \& Lima, L. M. T. R. Structural meta-analysis of regular human insulin in pharmaceutical formulations. Eur. J. Pharm. Biopharm. 85, 1112-1121 (2013).

51. Luthra, A. et al. Human Antibody Bispecifics through Phage Display Selection. Biochemistry 58, 1701-1704 (2019).

52. Ostrov, D. A., Shi, W., Schwartz, J. C., Almo, S. C. \& Nathenson, S. G. Structure of murine CTLA-4 and its role in modulating T cell responsiveness. Science 290, 816-819 (2000). 
Fig. 1: Mechanisms of protein therapeutics and anti-CRISPR proteins.

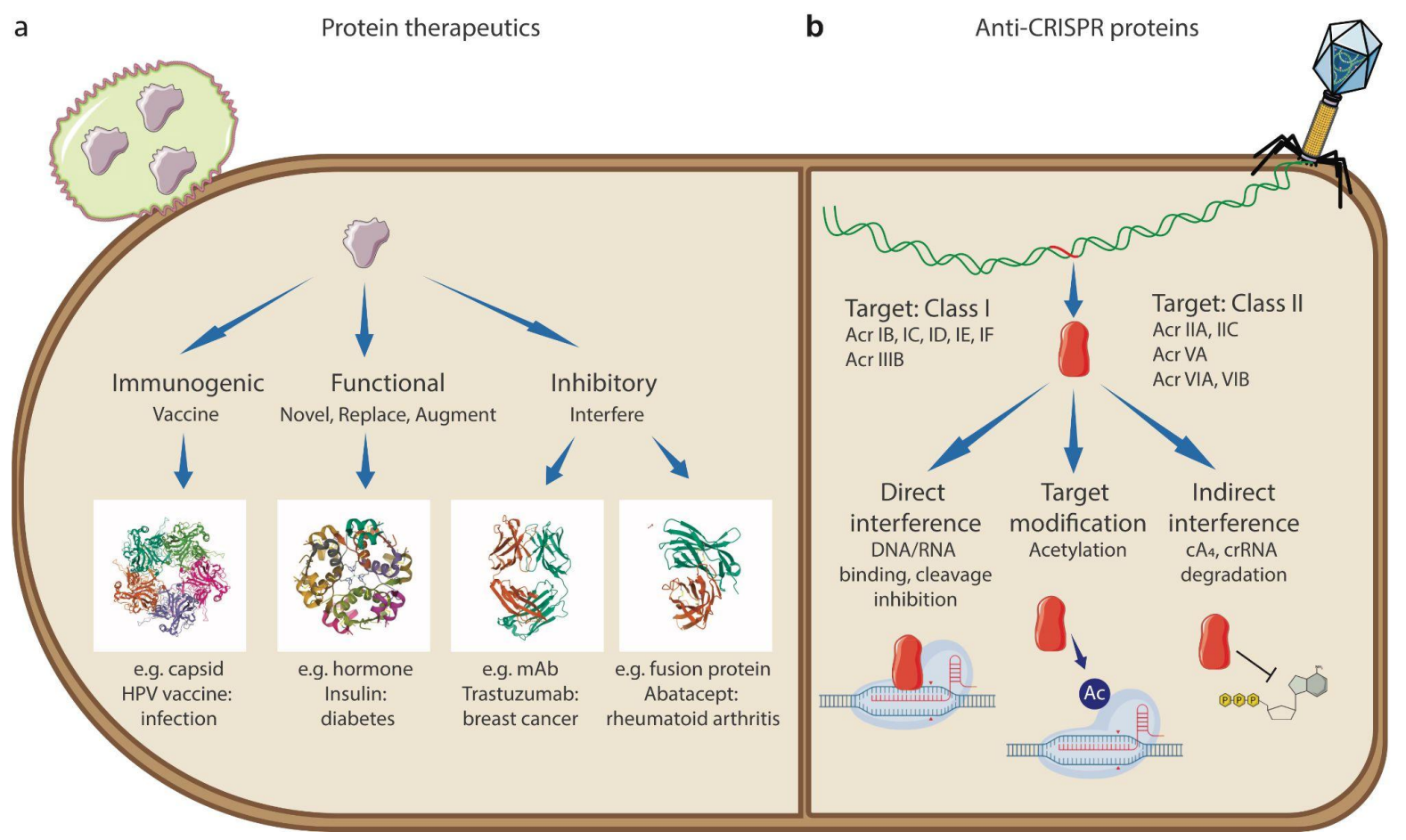

a, Mechanism of protein therapeutics. The first group consists of prophylactic or therapeutic vaccines to develop immunity against foreign or cancer cells. A highly successful protein vaccine against human papillomavirus (HPV) combining the capsids from four pathogenic HPV strains is given as an example (PDB: 2R5K ${ }^{49}$ ). The second group consists of protein therapeutics that provide novel functions, replace deficient or abnormal proteins, or augment existing activities. The approval of recombinant insulin in the 1980s to treat diabetes as the first abundant, inexpensive and low immunogenic therapeutic protein is given as an example (PDB: $4 \mathrm{~F} 8 \mathrm{~F}^{50}$ ). The third group consists of proteins that interfere with target proteins by high binding specificity. Some monoclonal antibodies use antigen recognition sites or receptor-binding domains like Trastuzumab against breast cancer cells (PDB: $6 \mathrm{MH} 2{ }^{51}$ ). Some fusion proteins inhibit target proteins by blocking interaction sites like Abatacept against rheumatoid arthritis (PDB: 1DQT ${ }^{51,52}$ ) b, Mechanisms of anti-CRISPR proteins. Upon successful infection, phage genomes express anti-CRISPR proteins that neutralize host CRISPR-Cas immunity. Anti-CRISPR proteins target various types of both Class I and Class II CRISPR-Cas systems and the inhibitory mechanisms are highly diverse, including direct interference of DNA/RNA binding and cleavage of Cas complexes, enzymatic inhibition of the active site by acetylation, and nuclease activity of degrading the crucial signaling molecule (cyclic nucleotide $\mathrm{cA}_{4}$ ). 
Fig. 2: Performance analysis of AlphaFold on anti-CRISPR proteins in comparison to the CASP14 dataset.

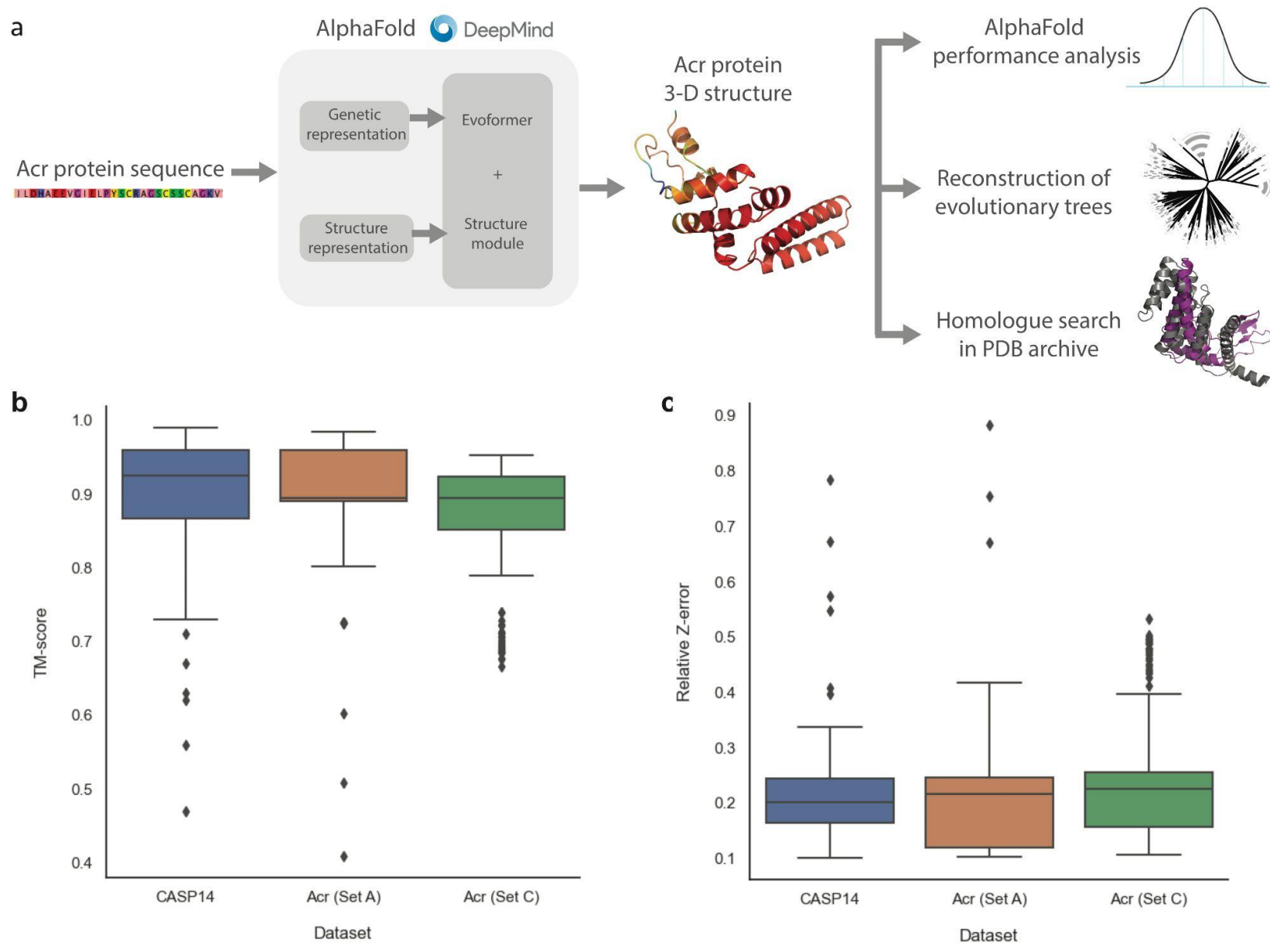

a, Overall workflow to analyze the 3-D macromolecular structures of Acr protein sequences predicted with AlphaFold. b, The performance of AlphaFold on the Acr protein datasets in comparison to the CASP14 dataset using TM-scores. The closer the TM-score is to 1, the more similar the predicted structure is to its true experimental structure. c. The performance of AlphaFold on the Acr protein datasets in comparison to the CASP14 dataset using relative Z-errors. The closer the relative Z-error is to 0 , the more similar the predicted structure is to its true experimental structure. (Set A: Verified Acr proteins with experimental structures, Set C: Putative Acr proteins with experimental structures). 
Fig. 3: Evolutionary trees of anti-CRISPR proteins.
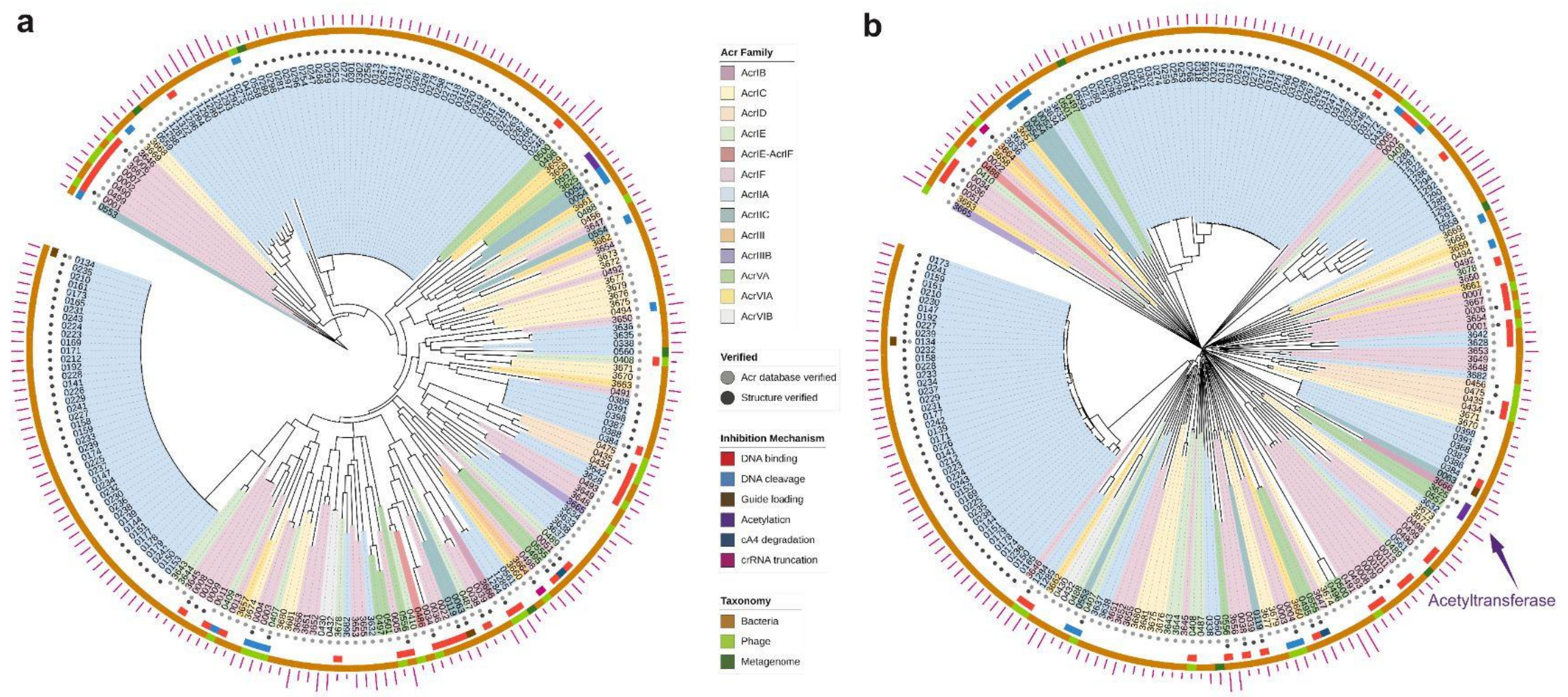

a, The phylogenetic tree of anti-CRISPR proteins reconstructed using sequence-based methods (Set A + Set B; $n=207$ ). $\mathbf{b}$, The structural tree of anti-CRISPR proteins reconstructed using structure-based methods (Set A + Set B; $n=207$ ). The clades of the two evolutionary trees were coloured by the Acr Family. The inner to outer rings display the Acr verification status, structural verification status, inhibition mechanism and source organism taxonomy. The outer magenta bars represent the genetic sequence length of each protein. 
Fig. 4: AlphaFold-predicted 3-D structures of outlier anti-CRISPR proteins.

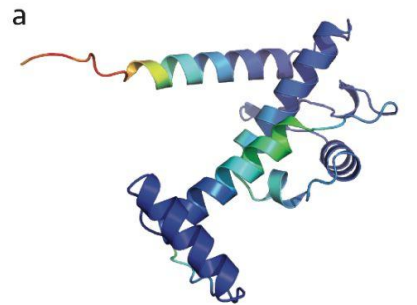

AlphaFold: 3674 (AcrlC6) pLDDT score: 87.14

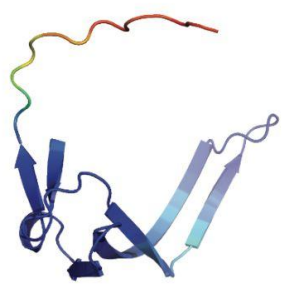

AlphaFold: 3658 (AcrVIA2) pLDDT score: 86.62

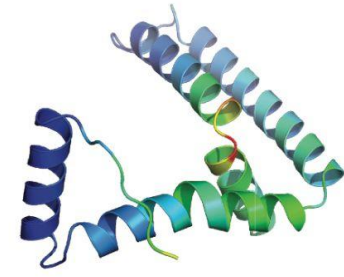

AlphaFold: 3638 (AcrllA19) pLDDT score: 90.77

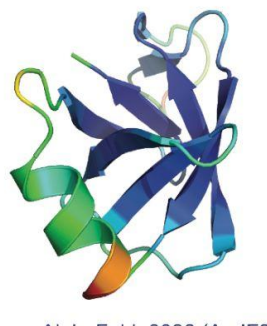

AlphaFold: 0036 (AcrlF8) pLDDT score: 91.53

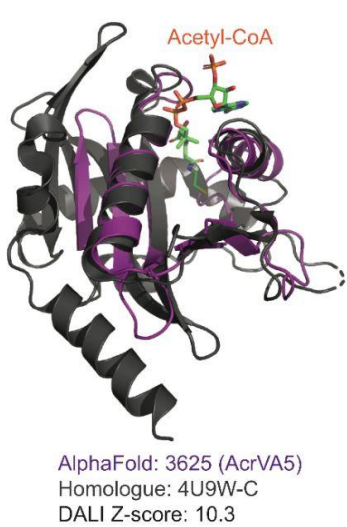

pLDDT:

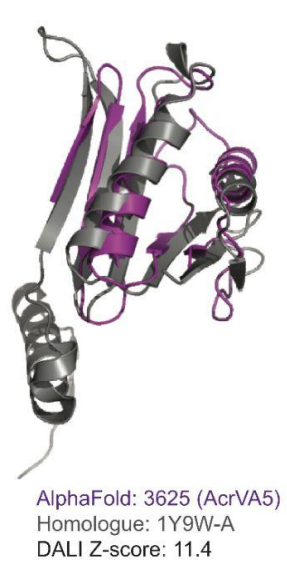

a, The AlphaFold-predicted structures of the Acr proteins without homologues in the Protein Data Bank archive (Dali Z-score $<2$ ). The 3-D protein structures are coloured according to the b-factor spectrum in PyMol, with a per-residue estimate of the AlphaFold confidence on a scale from 0 - 100 (high pLDDT accuracy in blue, low pLDDT accuracy in red). b, Superimposition of the AlphaFold-predicted structures of Acr proteins and their closest structural homologues retrieved from the Protein Data Bank archive. All the closest structural homologues in grey have functional annotations related to acetyltransferase. The Acr labelled ' 3625 ' has a cofactor (acetyl-CoA) bound, revealing the functionally critical site of the enzyme. c, Functional analysis of the closest homologues to the AlphaFold-predicted Acr proteins without experimental structures (Set B). Only the functional annotations above the significance threshold of Dali Z-score $(>4)$ were included. 
Table1. Closest homologue (highest Dali Z-score) to the AlphaFold-predicted structure of Acr proteins with acetyltransferase annotations.

\begin{tabular}{|c|c|c|c|c|c|c|c|c|}
\hline Acr ID & Family & Type & Length & Homologue & Dali Z-score & Annotation & \%ID structure & \%ID sequence \\
\hline 3625 & AcrVA5 & V-A & 92 & 4 U9W-C & 10.3 & N-Alpha-Acetyltransferase & 14 & 6.8 \\
\hline 3625 & AcrVA5 & V-A & 92 & 1 1Y9W-A & 11.3 & Acetyltransferase & 22 & 16.8 \\
\hline 3666 & AcrIB & I-B & 193 & 7AK8-B & 16.8 & Acetyltransferase & 21 & 17.6 \\
\hline
\end{tabular}

\%ID structure: percentage identity in structure

\%ID sequence: percentage identity in sequence 